Rapport - Société canadienne d'histoire de l'Église catholique

\title{
Les Filles de Mère d'Youville dans la région de Sudbury
}

\section{Sainte-Monique-d'Ostie}

Volume 27, 1960

URI : https://id.erudit.org/iderudit/1007405ar

DOI : https://doi.org/10.7202/1007405ar

Aller au sommaire du numéro

Éditeur(s)

La Société canadienne d'histoire de l'Église catholique

ISSN

0318-6148 (imprimé)

1927-7075 (numérique)

Découvrir la revue

Citer cet article

Sainte-Monique-d'Ostie (1960). Les Filles de Mère d'Youville dans la région de Sudbury. Rapport - Société canadienne d'histoire de l'Église catholique, 27, 83-91. https://doi.org/10.7202/1007405ar

Tous droits réservés @ La Société canadienne d'histoire de l'Église catholique, 1961
Ce document est protégé par la loi sur le droit d'auteur. L’utilisation des services d'Érudit (y compris la reproduction) est assujettie à sa politique d'utilisation que vous pouvez consulter en ligne.

https://apropos.erudit.org/fr/usagers/politique-dutilisation/ 


\section{Les Filles de Mère $d^{\prime} Y$ Youville dans la région de Sudbury}

Je serais tentée de commencer cette petite causerie sur les origines des fondations des Sours Grises dans la région par cet axiome connu des historiens comme des amateurs de vieilles chroniques communautaires: l'histoire est un perpétuel recommencement.

Oui, l'histoire se répète, qu'elle soit jouée sur l'échiquier mondial, national ou simplement local ! L'actualité, il est vrai, lui confère un certain imprévu, mais comme, à distance, tout se tasse dans à peu près les mêmes formules, il n'y a souvcnt que les dates à changer aux étiquettes.

Ainsi, l'apostolat des Sœurs Grises, dans la région de Sudbury pourrait être résumé par quelques mots clés: pauvreté, pénurie de main-d'œuvre, travail consciencieux et persévérant, patriotisme, ténacité. Ce dernier mot mériterait d'être mis en lumière. Oui, certes, une ténacité merveilleuse, miraculeuse, une ténacité de lierre robuste s'agrippant, par toutes ses ramilles, par toutes ses fibrilles, au roc millénaire auquel il semble vouloir se fusionner. Et nous voici en pays de connaissance où même les symboles nous «observent avec des regards familiers ». A l'ombre de la croix salvatrice, le lierre des Sours Grises n'a-t-il pas su prendre fortement racine dans la bonne roche de Sudbury ?...

Relisons quelques pages de cette glorieuse épopée. Dans toutes les mémoires revivent les dernières phases d'un drame dont le dénouement heureux s'effectua au printemps de la présente année: je veux parler de l'installation de nos élèves du cours secondaire dans leur beau collège neuf, le Collège Notre-Dame. Or ce drame - le mot est à retenir - eut une longue préparation dans le passé, un passé bourrelé de luttes raciales, assombri par la pauvreté, l'ignorance et les multiples préjugés, un passé qui, puisque nous le considérons du point de vue de l'éducateur, dut faire front contre une coalition de forces supérieures par le nombre et par le prestige, un passé qui sortit vainqueur de l'enquête du docteur Merchant et du Règlement XVII et qui, marchant de progrès en progrès, se rendit le sûr garant d'un avenir plus viable et plus prometteur.

Au cours de notre enquête, je voudrais vous faire visiter, par la pensée, quelques lieux de "pèlerinage ", quelques monuments historiques dont bon nombre d'entre nous ignorent sans doute jusqu'à l'existence. J'entreprends donc un petit travail de vulgarisation, non pour l'ancienne garde qui a vécu cette ère glorieuse, mais plutôt pour la jeune génération, celle qu'on se plaît à appeler la génération montante et qui devrait, plus que toute autre, cultiver cette mémoire qui attache aux vieilles choses tout en formant le lien indispensable entre le passé et l'avenir. D'ailleurs, ceux que nous nommons les pionniers aimeront peut-être revivre ces 
heures difficiles auxquelles le recul du temps confère un charme nostalgique, presque mystique.

Allons en tout premier lieu frapper à la porte de notre hôpital Saint-Joseph et retournons plus de soixante ans en arrière. C'est ici que débuta le zèle des Sours Grises de la Croix. Appelées à Sudbury dès 1896, grâce au travail infatigable des RR.PP. Jésuites, les promoteurs de l'œuvre, elles entrèrent en 1899 dans leur hôpital Saint-Joseph, à l'emplacement qu'il occupe encore aujourd'hui. Chose assez curieuse,

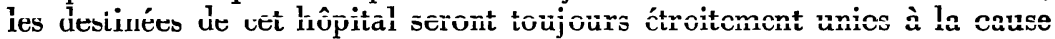
de l'enseignement. En effet, les religieuses enseignantes qui arrivent dès 1898 habiteront à l'hôpital. Pendant de longues années, elles soupireront comme les petits de la colombe pour le trou dans la pierre, pour leur " petit couvent " bien à elles, où elles pourront vaquer, loin de l'odeur de la naphthaline et des lentes agonies, à leur mission toute de jeunesse et d'espoir.

Mais cette heureuse prise de possession sera forcément retardée jusqu'en 1920, date de l'exode des sœurs institutrices vers le petit couvent, de la rue Beech. Ainsi, quarante ans plus tard, l'heure du départ sonnera de nouveau, cette fois pour les élèves du couvent Notre-Dame. Enfin, les essaims si longtemps éparpillés prendront leur vol vers les cimes, vers le spacieux asile qu'on leur aura aménagé sur les hauteurs du plateau Lebel, là où l'air est pur et les horizons, sans limites, là où même le roc revêt des tendresses de mère, une douceur d'aïeule débonnaire.

Mais avant de partir, nos étudiantes devront s'acquitter d'un pieux devoir: celui de remercier celles qui pendant neuf ans, leur auront accordé une si généreuse hospitalité. Hôpital, hospitalité ! Ces deux mots n'ont-ils pas la même origine... Nos sœurs hospitalières peuvent en effet revendiquer, hier comme aujourd'hui, leur légitime part dans l'éducation de la jeunesse canadienne-française de Sudbury.

Notre prochain arrêt se fera sur la colline, puisque nous y sommes rendus. Nous nous arrêterons donc devant un vieil édifice en briques rouges, construction peu esthétique mais solide, cubulaire même. Vous l'avez reconnu: c'est notre cher orphelinat d'Youville, le premier monument dressé à l'éducation canadienne-française dans la ville. En effet, avant d'être voué aux œuvres de charité, cet édifice hébergea dès 1898 , nos petits Sudburiens. Sœur Marie-Gertrude, une ouvrière de la toute première heure, s'y dévoua pendant de nombreuses années, en collaboration avec plusieurs institutrices laïques et religieuses. Il est intéressant de noter ici que la toute première classe fut ouverte au deuxième étage du presbytère de la paroisse Sainte-Anne. Mais cela, c'est dans les premiers temps de la colonie, avant l'ère des Sœurs Grises.

En 1909, l'école catholique devenue trop petite, le père Lefebvre, s.j., sacrifia une partie de la salle paroissiale (actuellement l'édifice de la pharmacie Michaud) et y installa deux classes. En puisant aux sources orales, j'eus la bonne fortune d'apprendre que la supérieure actuelle du collège Notre-Dame, sœur Agnès-de-Jésus, enseigna toute jeune fille, en ces temps héroïques, dans une des classes de la salle du Jubilé. Le 
problème n'était pas l'exiguïté des locaux, bien au contraire ! Il lui fallut se contenter d'une vaste salle de théâtre où les soixante marmots de première année auraient pu s'ébattre tout à leur aise si une autorité vigilante ne les en eut empêchés.

Ce ne sera qu'en 1915 que l'Ecole centrale, celle que nous connaissons encore aujourd'hui sous ce vocable ancien, ouvrira toutes grandes ses portes à une population enfantine qui paraît vouloir faire éclater les cadres. Nos vieilles chroniques doivent se contenter de consigner, d'une année à l'autre, lors de l'ouverture des classes, les mêmes explosions de la population scolaire suivies des mêmes jérémiades professorales. Les classes sont bondées d'élèves, les maîtresses ne peuvent suffire à la tâche, on ne sait où mettre le surplus d'élèves. Ne suis-je pas tombée sur ce détail stupéfiant dans son laconisme: sœur Saint-Cyriaque accueillit en sa classe de première année, en septembre, 1910, une sarabande de cent vingt marmots accourus aux sources du savoir. Vous imaginez la scène! Cent vingt petites têtes blondes ou brunes, deux cent quarante petits yeux inquisiteurs ou larmoyants, et toutes ces mamans attendries encombrant classes, corridors et se déversant sans doute sur les flancs de la montagne.

Et les chroniques n'ajoutent plus une ligne à l'étrange odyssée de sœur Saint-Cyriaque. Espérons tout de même que la situation se sera sensiblement améliorée lors de la fameuse enquête du docteur Merchant, deux ans plus tard.

Malgré tout, la première école bilingue tint bon jusqu'en 1929, alors qu'elle passa aux Sœurs Grises de Nicolet et devint l'orphelinat d'Youville. Pour obvier à cette perte du côté scolaire, de nouvelles écoles se fondent: Nolin, Sainte-Marie et Saint-Albert. Mais en septembre, 1929, on répète les mêmes doléances: l'espace manque partout. Pour donner une idée de l'acuité du problème: trois classes doivent s'ouvrir à la salle paroissiale, une autre au sous-sol de l'église SaintJoseph, une autre dans la salle des maîtresses à l'Ecole centrale, une dernière dans la bibliothèque à l'école Sainte-Marie. Deux des nôtres ont déjà magistralement analysé cette époque troublée: sœur SaintIrénée, ancienne directrice des écoles à Sudbury, lors d'une conférence prononcée en 1945, devant la Société historique du Nouvel-Ontario, et le regretté docteur Raoul Hurtubise, un des champions de nos écoles bilingues. Nous renvoyons les intéressés à ces deux précieux documents de la Société historique, si riches en leçons et en avertissements salutaires.

Mais nous voici rendus en 1930. Date mémorable dans l'histoire de notre enseignement secondaire. En septembre de cette année, sœur Paul-Emile ajoute au cinquième cours bilingue une classe de Middle School et d'Upper School. Elle établit ses quartiers généraux dans une classe de l'Ecole centrale. Les élèves d'alors rendent encore hommage à sa vaste compétence, à son savoir-faire. Sœur Saint-Lorenzo lui succède bientôt et le bon travail se poursuit auprès de la jeune élite. Mais, en 1939, s'abat la catastrophe. Comme l'Ecole centrale ne suffit plus pour les cours primaires, la Commission scolaire décide de sup- 
primer les classes du cours supérieur et de les transférer à la Haute Ecole.

C'est alors que surviendra ce que le R.P. Belcourt a appelé: "le grand vide de 1939 à 1948, "véritable tragédie dont les suites se firent longtemps sentir. Mais les notables de la ville, âmes généreuses et profondément patriotiques, ne se tinrent pas battus pour si peu. Les citoyens vont en délégations nombreuses auprès des autorités. Il est intéressant de suivre ce travail en profondeur dans nos vieilles chroniques. Des noms aux résonances familières $s^{\prime} y$ font jour: les Pilouffe. les Hurtubise, les Ricard, les Lapalme et combien d'autres! Les contıadictions foisonnent, les incompréhensions se multiplient, mais rien ne semble dérouter ces âmes chevaleresques. Et après bien des cheminements obscurs, bien des reculs déconcertants, la divine Providence arrive à ses fins. Notre collège actuel n'en est-il pas le plus vibrant témoignage ?...

Il convient d'évoquer ici le souvenir de quelques conseillers prudents et sages qui firent beaucoup pour l'œuvre des écoles bilingues dans Sudbury, commissaires et inspecteurs dont nos annales ont gardé le souvenir. Nommons en passant $M$. Laurier Carrière qui réussit à amener des Sœurs Grises à Hanmer, M. Rémi Millette, aux attentions toutes paternelles, M. Paul-Eudore Piché, l'homme de système et le psychologue averti, M. Roland Lalonde, un défenseur de la langue française et promotteur de ses droits. Parmi les commissaires d'écoles combien d'ouvriers laborieux. L'un d'entre eux, M. Félix Ricard, s'est vu honoré, l'an passé, par le Conseil de la Vie française. Mais combien d'autres ont travaillé dans l'ombre, ont lutté vaillamment sans remporter de couronne! Rendons-leur du moins aujourd'hui le témoignage public de notre reconnaissance. L'occasion ne s'y prête-t-elle pas magnifiquement alors que nous goûtons, en ce bel auditorium neuf, à la réalisation d'un noble rêve?

Ce collège, dès 1920 , on le désirait ardemment, mais dans ces temps anciens, tout le monde disait: le pensionnat! Les sours institutrices venaient de quitter l'hôpital. Il fallait leur trouver un asile convenable au bas de la côte. Les circonstances les obligèrent d'abord de se contenter d'un petit logis provisoire, à l'angle de la rue Beech et Notre-Dame, où se logea longtemps la librairie Loisirs. Mais le mot est lâché: "On rêve d'un pensionnat !...» $L a$ phrase se trouve telle quelle dans nos anciennes chroniques. Rêve de longue haleine puisqu'il ne deviendra réalité qu'en 1960, quarante ans plus tard. Et nous citons textuellement quelques lignes des chroniques de 1922.

Où bâtir ? Voilà la question. Les opinions sont partagées au sujet du site. Notre révérende Mère choisirait le terrain où se trouve l'ancienne école (aujourd'hui le Foyer d'Youville) ou sur la côte, en arrière.

Cette fin de phrase n'a-t-elle pas quelque chose de vieillot, de charmant, mais en même temps, de sagement prophétique? Ne manifeste-t-elle pas clairement la puissance de vision de cette maîtresse-femme de jadis, rotre vénérée mère Saint-Albert? 
Mais il faudra attendre l'âge atomique avant de pouvoir escalader les cimes du plateau Lebel. Entre temps, on tente un compromis. On quitte bientôt le minable couvent sur la rue Beech pour habiter l'ancienne demeure du juge Mulligan, sise à 72 , rue Louis. Les sœurs sont heureuses. La maison est spacieuse, la rue beaucoup moins bruyante. Et la Providence veille...

Et nous voici rendues à notre troisième lieu de pèlerinage; le petit couvent qui, depuis que nous l'avons quitté, pour notre collège, l'année dernière, s'est acquis l'affectueuse appelation du ... "Petit couvent au bas de la côte."

Ce couvent qui est en train de se faire rénover complètement sous la direction experte de la supérieure actuelle, bâtisseuse de premier plan, ce couvent avec ses marches vermoulues, son toit français, son balcon décoratif et son annexe moderne, constitue, à lui seul, un authentique témoin des temps révolus. Pendant quarante ans, il demeura la cellule vitale de la communauté des sœurs enseignantes de Sudbury.

Comme tout organisme fait pour durer, ce couvent vécut une histoire assez mouvementée. Sans cesser de demeurer la résidence des sœurs enseignantes de Sudbury, il servit tour à tour de pensionnat pour les jeunes filles, de foyer pour les institutrices laïques, et prêta des salles de classe pour les élèves du cours secondaire.

En 1929, les autorités jugent nécessaire d'acheter le terrain voisin du couvent et on confie, à un architecte de la ville, la construction d'une vaste allonge formant maintenant le bâtiment central de l'édifice. Nous lisons dans les chroniques:

Deo gratias! Le creusage pour les fondations de notre Couvent, c'est-à-dire, l'agrandissement, a commencé à onze heures vingt-cinq.

Onze heures vingt-cinq. Pas tout à fait la demie ! Pour nous qui avons vécu les heures inoubliables de mai 1959 et qui avons vu lever la pelletée de terre par le maire Fabbro, à l'emplacement même de notre présent collège, pour nous qui avons vu un rêve de plusieurs générations prendre corps avec chaque poutre d'acier érigée, cette joie délirante est fort compréhensible et très légitime.

Mais avec les années, même cette annexe s'avérera trop petite. Les sœurs se mettront à l'étroit. Sur ce chapitre, les religieuses présentes qui ont vécu ces années héroïques en auraient long à dire. En 1948, le couvent décide d'ouvrir ses portes aux jeunes filles désireuses de poursuivre leurs études secondaires. Afin d'accommoder les élèves, les trente-trois sœurs devront forcément vivre à l'étroit. Bientôt, le réfectoire sera transformé en salle de classe; les sœurs prendront leurs repas dans la cuisine et la vaisselle sera lavée sur un radiateur dans le corridor. Les plus jeunes s'assoiront sur un petit banc en long pourvu d'un solide dossier: le mur de l'appartement. Se lever pendant les repas sera inviter à la catastrophe. Il faudra attendre que tout le monde ait fini! Et la cuisinière, où se logera-t-elle? Ah ! que la fatalité pèse lourdement sur la cuisinière d'alors! Avec son poêle et ses marmites, elle sera reléguée dans une minuscule cuisine d'été exposée aux quatre vents, au 
plancher mal étayé, antiquité vénérable qui a réussi à se maintenir debout jusqu'à ce jour mais qui devra s'affaisser et disparaître bientôt sous la hache des démolisseurs.

Une autre antiquité dont les jours sont comptés aussi, et qui connut ses années d'épopée, c'est la petite maison en bois, à la charpente fatiguée, au teint jaune, bilieux même, que vous pouvez visiter si vous aimez les vieilles choses, au numéro 84 , de la rue Louis. Cette maison à l'apparence souffreteuse sut ouvrir ses portes grinçantes à la joyeuse rihamhelle des netits du jardin d'enfants. En 1949, elle reçut les élèves de la $9^{e}$ année du pensionnat Notre-Dame-du-Bon-Conseil. Je me souviens que le professeur d'anglais d'alors, sœur Jacques-Marie, avec qui je faisais la rotation, avait fait écrire en belles lettres gothiques sur toute la longueur de son tableau de côté: "It's not the house that makes the home but the people who live in it !»

Entre temps, les pèlerinages se font nombreux sur la montagne. On m'a raconté quelques-unes de ces secrètes équipées où subrepticement, les sœurs, conduites par mère Saint-André-Corsini, supérieure générale d'alors, allaient déposer médailles et reliques dans les crevasses des rochers sympathiques, espérant contre tout espoir, semant sur la roche aride le grain paradoxal de leurs plus riches espérances. Et la moisson germa, malgré tout. Elle se leva, forte et drue et nous espérons qu'elle saura produire le centuple promis aux âmes laborieuses et de bonne volonté.

Mais en 1947, les autorités n'étaient pas encore décidées sur l'emplacement du futur externat. Nous pouvons intercaler ici l'ardente croisade du R.P. Migneault, s.j., dont le mot d'ordre était: "La côte pour la jeunesse! Les jeunes pour la côte !» Il voulait pour cette portion choisie de son troupeau la pureté des sommets, l'élan des cimes et la noblesse des grands horizons.

Le souvenir du vénérable Jésuite rappelle à notre mémoire d'autres noms de saints prêtres qui se dépensèrent sans compter sur l'œuvre commune et qui surent apporter à nos sœurs l'appui de leur science et de leur longue expérience. Rappelons l'activité apostolique du R.P. Rosaire Legault, s.j., le patriotisme éclairé du R.P. Alphonse Deguire qui prit la cause de notre pensionnat à cœur et fit plusieurs démarches dans ce but; rappelons les touchantes exhortations du R.P. Joseph Gamache, la sympathie du bon père Comte, spectateur assidu de toutes nos séances scolaires. Mais revenons à notre collège qui au temps du père Mignault n'était qu'un rêve lointain et, à certain moments, un projet des plus inconsistants et des plus chimériques.

Mais voici qu'en septembre 1949, les difficultés semblent aplanies et l'excavation commence sur le plateau Lebel. Mais les terribles détonations de dynamite ont à peine éteint leurs réverbérations que les travaux cessent. Toute construction doit être suspendue pour un temps indéterminé: le problème financier a revêtu des proportions inquiétantes. Ainsi, pendant de longues années, l'excavation demeurera comme une plaie béante ouverte à tous les cieux, plaie qui a laissé ses cicatrices dans les âmes des vaillantes pionnières de la toute première heure. 
Il conviendrait de nommer ici la principale instigatrice du projet, celle qui sut engager une lutte tenace contre des oppositions formidables et sans cesse renaissantes: la révérende mère Saint-Maximilien. L'œuvre a pu longtemps bénéficier de son appui et de ses sages directives. Il nous est doux de lui rendre ce soir cette expression publique de notre estime et de notre reconnaissance.

Mais comme rien ne bouge sur la montagne, on se voit contraint, en 1956, de faire construire un immeuble temporaire en briques jaunes où s'ouvrent bientôt trois belles et larges classes. Mais qu'est-ce que trois classes alors qu'il nous en faudrait vingt? Ainsi, maîtresses et élèves devront jusqu'en 1960 faire contre mauvaise fortune, bon coeur. En plus des trois classes ci-haut mentionnées, une classe continuera de se loger au couvent, tandis que les élèves des onzième, douzaine et treizième années seront cantonnées en d'anciennes chambres de malades, dans une aile désaffectée de l'hôpital. J'aurai eu le privilège d'enseigner, pendant une couple d'années, dans une de ces classes toutes sur la longueur, aux rangées inexistantes puisque les pupitres seront groupés quatre par quatre, au petit tableau grand comme la main au-dessus de la tribune, aux lumières fluorescentes presque au niveau des yeux du professeur... Détail entre mille qui est pourtant resté gravé dans les mémoires: un seul voyage au tableau de côté suffisait à faire tomber toutes les brosses et tous les bouts de craie de leur précaire perchoir.

Malgré tout, la joie règne dans les locaux éparpillés et un bon travail s'y fait. Parents, élèves et institutrices consentent généreusement aux sacrifices exigés. Grâce au dévouement inlassable d'une directrice hors série, j'ai nommé sœur Claire-de-Marie, qui dirige les destinées du collège depuis bientôt onze ans, cette institution entre résolument dans une ère de progrès. Les graduations deviennent chaque année de plus en plus nombreuses. Soit qu'elles se dévouent comme institutrices, gardes-malades, mères de famille, bachelières ou filles de bureau, nos anciennes savent faire honneur à la formation reçue et leur Alma Mater, dans toute sa récente splendeur, peut se dire fière de cette vigoureuse relève.

Notre petit pèlerinage dans le passé touche à son terme. Mais avant de nous quitter, retournons une dernière fois au couvent au bas de la côte, au petit couvent de la rue Louis. Pendant longtemps, cette maison de briques rouges portera l'appellation de "Maison Mère du Nord ». En effet, combien de fois n'a-t-elle pas accueilli d'insignes visiteuses ? Combien de fois n'a-t-elle pas servi de pied-à-terre aux religieuses des missions environnantes? Dès 1922, nos Chroniques consignent ces visites fraternelles. On vient de partout, de Chelmsford, car la mission existe depuis 1906, de Verner, fondé en 1917, de Noëlville, qui date de 1936, de Hanmer, de Cartier où nos sœurs besognent depuis 1940. A certains temps la famille est grande! Certaines voyageuses devront même quelques fois rebrousser chemin et gravir la côte vers l'hôpital. Mais ce ne sera certes pas manque de bonne volonté de la part du vieux couvent aux multiples fonctions, aux murs trop étroits pour sa nombreuse nichée. 
Après une carrière si remplie, on pourrait croire que l'histoire du couvent serait close. Pas du tout ! Depuis le début de l'été, il semble vouloir faire peau neuve, tout fier qu'il est d'avoir été choisi comme Maison provinciale pour la province Sainte-Marie, sous la direction de la provinciale actuelle, mère Ange-Gabriel.

Mais petit à petit, la ruche se vide. L'essaimage reste à l'ordre du jour. Après le départ massif des sœurs pour le cours secondaire, deux autres départs se sont effectués pour les nouvelles fondations de NotreDame-de-Fatima de B̈lezard Vailiey et pour le couvent des Saints-Ánges de la paroisse Saint-Eugène de Sudbury. Les abeilles s'envolent, c'est la loi de la nature; mais la vieille ruche garde le parfum des vieilles choses et retentit encore du musical bruissement des beaux jours passés. Peut-être même conserve-t-elle encore, dans son vieux cœur cinquantenaire, certains noms qui font sa gloire aujourd'hui: une ex-supérieure générale, présentement provinciale aux Etats-Unis: mère Saint-AndréCorsini, deux directrices générales des écoles, subséquemment provinciales: mère Sainte-Cécilia et mère Saint-François, sans oublier sœur Saint-Irénée que nous avons parmi nous ce soir. Comment passer sous silence le nom de celle qui dirige la chorale aujourd'hui et qui pendant dix-sept années consécutives, se dépensa auprès de la population étudiante de Sudbury? Vous avez tous reconnu sœur Jeanne-Mance! N'a-t-elle pas maintes fois fait valoir sa devise: "Toujours victorieuse, jamais battue! » dans les nombreux festivals de chant où se signalèrent ses élèves?

Et puis, tant d'autres vaillantes ouvrières! Scur Jeanne-Leber, une des fondatrices de l'école secondaire, sœur Berthe-Hélène qui prépara plusieurs lauréats au concours de français, lauréats qui surent lui faire honneur, entre autres, Mlles Anita Chevrier et Cora Ladouceur. Je ne puis terminer sans nommer une dernière fois notre Supérieure actuelle qui remplit présentement son troisième mandat comme supérieure dans cette région. Trois termes, c'est comme dans l'histoire des présidents américains, une chose assez rare... Que son humilité nous permette de le signaler $! . .$.

J'ai essayé, dans ce travail, de vous faire apprécier quelques institutions de notre ville. Aussi, si vous êtes un peu sentimental et un brin poète, ne passez pas indifférents devant le petit couvent de la rue Louis. Arrêtez-vous un instant, ne serait-ce que le temps d'un Gloria Patri, en attendant de traverser la rue qui la sépare de l'église SainteAnne. Et là, révérencieusement, invoquez les ombres des sœurs qui l'ont habité, qui s'y sont dévouées, qui l'ont quitté peut-être avec peine et qui y reviennent encore par la pensée. Vous pourrez plus facilement alors mesurer la distance qui sépare les courageuses aspirations du passé, des nobles réalisations du présent. Peut-être pourrez-vous même entrevoir dans la lumière qui descend de la montagne, les ambitieuses réalisations des générations futures, les sommets lumineux où doit accéder notre minorité catholique et française dans cette région du Nouvel-Ontario. 
Car l'avenir est aux gens de Sudbury, de cette besogneuse ville au front de granit et aux pieds nickelés. Aussi, comment ne pas terminer cette causerie par ces versets tirés des saints Cantiques et qui revêtent, dans l'occurrence, toute leur actualité.

Ceux qui se confient dans le Seigneur seront fermes comme la montagne de Sion; celui qui demeure dans Jérusalem ne sera jamais ébranlé.

Comme les montagnes entourent et défendent Jérusalem, ainsi le Seigneur environne les siens, aujourd'hui et pour toujours...

Sœur SAINTE-MoniQue-D'Ostie, s.g.c. Collège Notre-Dame, Sudbury, Ont. 